\title{
Homotopy classes of proper maps out of vector bundles
}

\author{
Thomas O. Rot
}

\begin{abstract}
In this paper, we classify the homotopy classes of proper maps $E \rightarrow \mathbb{R}^{k}$, where $E$ is a vector bundle over a compact Hausdorff space. As a corollary, we compute the homotopy classes of proper maps $\mathbb{R}^{n} \rightarrow \mathbb{R}^{k}$. We find a stability range of such maps. We conclude with some remarks on framed submanifolds of non-compact manifolds, the relationship with proper homotopy classes of maps, and the Pontryagin-Thom construction.
\end{abstract}

Mathematics Subject Classification. 55P57, 55Q10, 55Q45, 55Q55, 55N22.

Keywords. Proper homotopy theory, Pontryagin-Thom construction, Framed cobordism.

1. Introduction. A continuous map $f: X \rightarrow Y$ is called proper if $f^{-1}(C)$ is compact for all compact subsets $C$ of $Y$. A homotopy of proper maps is a homotopy $F:[0,1] \times X \rightarrow Y$ such that $F$ is a proper map. The assumption that a homotopy is a homotopy of proper maps is stronger than the assumption that the homotopy is homotopy through proper maps, i.e. the assumption that the maps $F_{t}: X \rightarrow Y$ are proper for every $t \in[0,1]$. A simple example of a homotopy through proper maps that is not a homotopy of proper maps is the map $F:[0,1] \times \mathbb{R} \rightarrow \mathbb{R}$ defined by $F(t, x)=t x^{2}+x$. To see this, note that the sequence $\left(\frac{1}{n},-n\right)$ is unbounded, but $F\left(\frac{1}{n},-n\right)=0$. This example is closely related to the compactness issues discussed in [5].

We denote by $[X, Y]$ the set of (unbased) homotopy classes of maps from $X$ to $Y$ and with $[X, Y]_{\text {prop }}$ the set of (unbased) homotopy classes of proper maps. For the set of homotopy classes of based maps between pointed spaces we write $\langle X, Y\rangle$.

In [1], we classified the homotopy classes of proper Fredholm maps of Hilbert manifolds into its model (real and separable) Hilbert space in terms of a suitable notion of framed cobordism. This classification uses an infinite dimensional and proper analogue of the Pontryagin-Thom collapse map, which is 
due to Elworthy and Tromba [3], see also the paper of Gęba [4]. The existence of the collapse map hinges on the fact that an infinite dimensional Hilbert space is diffeomorphic to the Hilbert space minus a point. This is of course not true for a finite dimensional vector space and the construction does not work in this setting. As we will discuss in Section 4, even though the framed cobordism class of a regular value is an invariant of the homotopy class of a proper map in the finite dimensional setting, the framed cobordism class is not able to distinguish all proper homotopy classes of proper maps into $\mathbb{R}^{k}$, nor do all framed submanifolds come from proper maps. Thus there does not exist a finite dimensional proper Pontryagin-Thom construction, which is why we are not able to compute $\left[E, \mathbb{R}^{k}\right]_{\text {prop }}$ for all open finite dimensional manifolds $E$ using a Pontryagin-Thom collapse map. In this paper, we are content with the classification of $\left[E, \mathbb{R}^{k}\right]_{\text {prop }}$ where $E$ is a real vector bundle over a compact Hausdorff space $M$. This classification does not use a Pontryagin-Thom collapse map.

Theorem 1.1. Let $E \rightarrow M$ be a normed real vector bundle over a compact Hausdorff space $M$. Denote by $S(E) \rightarrow M$ the associated sphere bundle of unit vectors. Then the set $\left[E, \mathbb{R}^{k}\right]_{\text {prop }}$ is in bijective correspondence with $\left[S(E), S^{k-1}\right]$.

We have the following corollary of Theorem 1.1 by taking $M$ to be a point and using the fact that based and unbased homotopy classes of maps from spaces to positive dimensional spheres coincide, cf. [6, Section 4A].

Corollary 1.2. $\left[\mathbb{R}^{n}, \mathbb{R}^{k}\right]_{\text {prop }}$ is in bijective correspondence with $\left[S^{n-1}, S^{k-1}\right]$. Thus if $n>1$ and $k>1$, we have that $\left[\mathbb{R}^{n}, \mathbb{R}^{k}\right]_{\text {prop }}$ is in bijection with $\pi_{n-1}\left(S^{k-1}\right)$. The set $\left[\mathbb{R}^{n}, \mathbb{R}\right]_{\text {prop }}$ has two elements if $n>1$ and four elements if $n=1$.

A proper map between non-compact and locally compact Hausdorff spaces extends to a continuous map between the one point compactifications by sending infinity to infinity. Similarly a homotopy of proper maps induces a homotopy in the one point compactification.

The one point compactification of a real vector bundle $E \rightarrow M$ over a compact Hausdorff space $M$ equals the Thom space $\operatorname{Th}(E)$ of the vector bundle and the one point compactification of $\mathbb{R}^{k}$ is homeomorphic to $S^{k}$ by stereographic projection. Thus we obtain a map $Q:\left[E, \mathbb{R}^{k}\right]_{\text {prop }} \rightarrow\left\langle\operatorname{Th}(E), S^{k}\right\rangle$. In Section 3, we show that the map $Q$ is bijective if $k$ is sufficiently large. If $E=\mathbb{R}^{n}$, the map $Q$ is nothing but the suspension $\pi_{n-1}\left(S^{k-1}\right) \rightarrow \pi_{n}\left(S^{k}\right)$ under the identification of $\left[\mathbb{R}^{n}, \mathbb{R}^{k}\right]_{\text {prop }}$ and $\pi_{n-1}\left(S^{k-1}\right)$ of Corollary 1.2.

For $l$ sufficiently large, the sets $\left[E \oplus \mathbb{R}^{l}, \mathbb{R}^{k+l}\right]_{\text {prop }}$ and $\left[E \oplus \mathbb{R}^{l+1}, \mathbb{R}^{k+l+1}\right]_{\text {prop }}$ are in bijection. Thus it makes sense to define the stable proper homotopy classes as

$$
\left[E, \mathbb{R}^{k}\right]_{\mathrm{prop}}^{S}=\lim _{l \rightarrow \infty}\left[E \oplus \mathbb{R}^{l}, \mathbb{R}^{k+l}\right]_{\mathrm{prop}},
$$

which are in bijection with the stable cohomotopy groups $\pi_{S}^{k}(\operatorname{Th}(E))$, cf. Corollary 3.2. Using Atiyah duality, we obtain the following result. 
Theorem 1.3. Let $M$ be an m-dimensional compact manifold with boundary $\partial M$. Let $E$ be the normal bundle of $M$ of some embedding of $M$ into $\mathbb{R}^{m+n}$. Then there is a bijection of $\left[E, \mathbb{R}^{k}\right]_{\text {prop }}^{S}$ with $\pi_{n+m-k}^{S}(M / \partial M)$.

In Section 4.4, we speculate on the classification problem in the case that $E$ is an arbitrary open manifold.

2. The proof of Theorem 1.1. For the remainder of the paper, $E \rightarrow M$ denotes a normed real vector bundle over a compact Hausdorff space $M$. The associated sphere and disk bundles of radius $R>0$ are

$$
S_{R}(E)=\{v \in E \mid\|v\|=R\} \quad \text { and } \quad B_{R}(E)=\{v \in E \mid\|v\|<R\} .
$$

We write $S(E)$ for $S_{1}(E)$ and $B(E)$ for $B_{1}(E)$.

Given a homotopy $F:[0,1] \times S(E) \rightarrow S^{k-1}$, we define the homotopy $P F:[0,1] \times E \rightarrow \mathbb{R}^{k}$ of proper maps by

$$
P F(t, v)= \begin{cases}\|v\| F\left(t, \frac{v}{\|v\|}\right), & v \neq 0, \\ 0, & v=0 .\end{cases}
$$

Compact subsets of $E$ are characterized as follows: A subset $K \subseteq E$ is compact if and only if it is closed and bounded. Here bounded means that $K \subseteq B_{R}(E)$ for some $R>0$. As $P F(\cdot, t)$ is norm preserving for all $t$, it follows that $P F$ is proper.

The same construction assigns to a map $f: S(E) \rightarrow S^{k-1}$ a proper map $P f: E \rightarrow \mathbb{R}^{k}$ and it therefore induces a map $P:\left[S(E), S^{k-1}\right] \rightarrow\left[E, \mathbb{R}^{k}\right]_{\text {prop }}$.

We will show that $P$ is bijective. Let us start with the injectivity. We need to show that $f_{0}$ and $f_{1}$ are homotopic if $g_{0}=P f_{0}$ and $g_{1}=P f_{1}$ are homotopic as proper maps. Let $G:[0,1] \times E \rightarrow \mathbb{R}^{k}$ be a homotopy of proper maps between $g_{0}$ and $g_{1}$. Then there exists an $R>0$ such that $G^{-1}\left(B\left(\mathbb{R}^{k}\right)\right) \subseteq$ $[0,1] \times B_{R}(E)$. It follows for any $v \in E$ with $\|v\|=R$ that $G(t, v) \neq 0$. The $\operatorname{map} F:[0,1] \times S(E) \rightarrow S^{k-1}$ given by

$$
F(t, x)=\frac{G(t, R x)}{\|G(t, R x)\|}
$$

is a homotopy between $f_{0}$ and $f_{1}$, hence $P$ is injective.

To show that $P$ is surjective, we need to show that, given a proper map $g: E \rightarrow \mathbb{R}^{k}$, there exists a homotopy of proper maps from $g$ to $P f$, where $f$ is some map $f: S(E) \rightarrow S^{k-1}$. As $g$ is proper, there exists an $R>0$ such that $g^{-1}\left(B\left(\mathbb{R}^{k}\right)\right) \subseteq B_{R}(E)$. The sphere bundle $S_{R}(E)$ is compact, hence there exists an $r \geq 1$ such that

$$
1 \leq\|g(v)\| \leq r, \quad \text { for all } \quad v \in S_{R}(E) .
$$

Consider the map $h: \mathbb{R}^{k} \rightarrow \mathbb{R}^{k}$ defined by

$$
h(x)= \begin{cases}x, & \|x\| \leq 1, \\ \frac{x}{\|x\|}, & 1 \leq\|x\| \leq r \\ \frac{x}{r}, & r \leq\|x\|,\end{cases}
$$


and define $g_{1}: E \rightarrow \mathbb{R}^{k}$ by $g_{1}(v)=h \circ g(R v)$. As $h$ is homotopic as proper map to the identity via $(t, x) \mapsto(1-t) h(x)+x$, it follows that the map $g_{1}$ is proper homotopic to $g$. Note that if $\|v\|<1$, then $\|R v\|<R$ and therefore $\|g(R v)\|<1$. We see that $g_{1}(B(E)) \subseteq B\left(\mathbb{R}^{k}\right)$ and similarly that $g_{1}(S(E)) \subseteq S^{k-1}$. Define $G_{1}:[0,1] \times E \rightarrow E$ by

$$
G_{1}(t, v)= \begin{cases}\frac{\left\|g_{1}(v)\right\|}{\left\|g_{1}\left((1-t) v+\frac{t}{\|v\|} v\right)\right\|} g_{1}\left((1-t) v+\frac{t}{\|v\|} v\right), & \|v\| \geq 1 \\ g_{1}(v), & \|v\| \leq 1\end{cases}
$$

The equation $\left\|G_{1}(t, v)\right\|=\left\|g_{1}(v)\right\|$ implies that $G_{1}^{-1}\left(B_{s}\left(\mathbb{R}^{k}\right)\right) \subseteq[0,1] \times$ $g_{1}^{-1}\left(B_{s}\left(\mathbb{R}^{k}\right)\right)$ for all $s$ and hence that $G_{1}$ is proper if $g_{1}$ is. Thus $g_{2}: E \rightarrow \mathbb{R}^{k}$, given by $g_{2}(x)=G_{1}(1, x)$, is a proper map that is proper homotopic to $g$. Let $f: S(E) \rightarrow S^{k-1}$ be the map obtained by restriction of $g_{2}$. Consider

$$
G_{2}(t, v)=(1-t) g_{2}(v)+t P f(v) .
$$

We want to prove that $G_{2}$ is proper. Note that $G_{2}$ sends $\overline{[0,1] \times B(E)}$ to $\overline{B\left(\mathbb{R}^{k}\right)}$ and $[0,1] \times(E \backslash B(E))$ to $\mathbb{R}^{k} \backslash B\left(\mathbb{R}^{k}\right)$. The map $\left.G_{2}\right|_{[0,1] \times B(E)}: \overline{[0,1] \times B(E)} \rightarrow$ $\overline{B\left(\mathbb{R}^{k}\right)}$ is proper as the domain is compact. We conclude that $G_{2}$ is proper if and only if

$$
\left.G_{2}\right|_{[0,1] \times(E \backslash B(E))}:[0,1] \times(E \backslash B(E)) \rightarrow \mathbb{R}^{k} \backslash B\left(\mathbb{R}^{k}\right)
$$

is proper.

For this, it is sufficient to show that for all $s>1$, there exists an $S>1$ such that $G_{2}^{-1}\left(B_{s}\left(\mathbb{R}^{k}\right) \backslash B\left(\mathbb{R}^{k}\right)\right) \subseteq[0,1] \times\left(B_{S}(E) \backslash B(E)\right)$. Note that for all $(t, v) \in[0,1] \times(E \backslash B(E))$, we have that

$$
G_{2}(t, v)=\left((1-t)\left\|g_{1}(v)\right\|+t\|v\|\right) f\left(\frac{v}{\|v\|}\right) \text {. }
$$

Consider all $(t, v) \in[0,1] \times(E \backslash B(E))$ such that $\left\|G_{2}(t, v)\right\| \leq s$. As $\left\|f\left(\frac{v}{\|v\|}\right)\right\|=$ 1 , this amounts to

$$
(1-t)\left\|g_{1}(v)\right\|+t\|v\| \leq s .
$$

Suppose the set of solutions of this equation is not contained in $[0,1] \times\left(B_{S}(E) \backslash\right.$ $B(E))$ for any $S$. Then we have a sequence $\left(t_{n}, v_{n}\right)$ of solutions such that $\left\|v_{n}\right\| \geq n$. Without loss of generality we take a subsequence such that $t_{n}$ converges to $t$ by the compactness of $[0,1]$. This subsequence will also satisfy $\left\|v_{n}\right\| \geq n$. If $t>0$, then there exists an $N$ such that for all $n \geq N$, we have $t_{n}>\frac{\bar{t}}{2}$ and

$$
\left\|v_{n}\right\| \leq \frac{1}{t_{n}}\left(\left(1-t_{n}\right)\left\|g_{1}\left(v_{n}\right)\right\|+t_{n}\left\|v_{n}\right\|\right) \leq \frac{2 s}{t},
$$

which contradicts the unboundedness of $v_{n}$. If $t=0$, then there exists an $N$ such that for all $n \geq N$, the sequence satisfies $t_{n}<\frac{1}{2}$ and

$$
\left\|g_{1}\left(v_{n}\right)\right\| \leq \frac{1}{\left(1-t_{n}\right)}\left(\left(1-t_{n}\right)\left\|g_{1}\left(v_{n}\right)\right\|+t_{n}\left\|v_{n}\right\|\right) \leq 2 s .
$$


The sequence $g_{1}\left(v_{n}\right)$ is therefore bounded and as the map $g_{1}$ is proper, it follows that the sequence $v_{n}$ is also bounded. This contradicts the assumption that $v_{n}$ is unbounded. This means that $\left.G_{2}\right|_{[0,1] \times(E \backslash B(E))}$ is proper. Thus $P f$ is proper homotopic to $g$ and $P:\left[S(E), S^{k-1}\right] \rightarrow\left[E, \mathbb{R}^{k}\right]_{\text {prop }}$ is surjective. We have already shown that $P$ is injective and Theorem 1.1 follows.

3. The one point compactification and stable (co)homotopy. Recall that the one point compactification of a non-compact, locally compact Hausdorff space $X$ is the space $X^{*}=X \cup\{\infty\}$ equipped with the following topology. All open sets $U$ of $X$ are declared open in $X^{*}$ along with all sets of the form $(X \backslash C) \cup\{\infty\}$ for all compact sets $C$ in $X$. Proper maps between non-compact, locally compact Hausdorff spaces induce continuous maps between the one point compactifications by imposing that $\infty$ is mapped to $\infty$. A homotopy of proper maps $F:[0,1] \times X \rightarrow Y$ induces a continuous map $F^{*}:([0,1] \times X)^{*} \rightarrow Y^{*}$. But $([0,1] \times X)^{*} \cong\left([0,1] \times X^{*}\right) /[0,1] \times\{\infty\}$. By the universal property of the quotient topology, we therefore also obtain a continuous map $F^{*}:[0,1] \times X^{*} \rightarrow Y^{*}$ which sends every $(t, \infty)$ to $\infty$. A homotopy of proper maps between unbased spaces is mapped to a based homotopy between the based spaces. The one point compactification of a vector bundle $E \rightarrow M$ over a compact Hausdorff space $M$ is called the Thom space of the bundle and we will write $\operatorname{Th}(E):=E^{*}$. Stereographic projection shows that $\left(\mathbb{R}^{k}\right)^{*} \cong S^{k}$ and in more generality, it holds that $\operatorname{Th}(E) \cong \overline{B(E)} / S(E)$. As was mentioned in the introduction, the map that forgets the basepoint induces a bijection between $\left\langle\operatorname{Th}(E), S^{k}\right\rangle$ and $\left[\operatorname{Th}(E), S^{k}\right]$ if $k \geq 1$. Thus from the one point compactification we obtain a $\operatorname{map} Q:\left[E, \mathbb{R}^{k}\right]_{\text {prop }} \rightarrow\left[\operatorname{Th}(E), S^{k}\right]$.

In our setting there are three suspension maps, which we all denote by $S$. To a map $g: S(E) \rightarrow S^{k-1}$ we associate the map $S g: S(E \oplus \mathbb{R}) \rightarrow S^{k}$ by

$$
S g(v, s)= \begin{cases}\left(\|v\| g\left(\frac{v}{\|v\|}\right), s\right), & v \neq 0 \\ (0, s), & v=0\end{cases}
$$

where we have normed the vector bundle $E \oplus \mathbb{R}$ via the formula $\|(v, t)\|=\|v\|+$ $|t|$. To a map $f: \operatorname{Th}(E) \rightarrow S^{k}$ we associate the map $S f: \operatorname{Th}(E \oplus \mathbb{R}) \rightarrow S^{k+1}$ via the same formula and to a proper map $f: E \rightarrow \mathbb{R}^{k}$ we associate the proper $\operatorname{map} S f: E \oplus \mathbb{R} \rightarrow \mathbb{R}^{k+1}$ by $S f(x, s)=(f(x), s)$. The following diagram is commutative

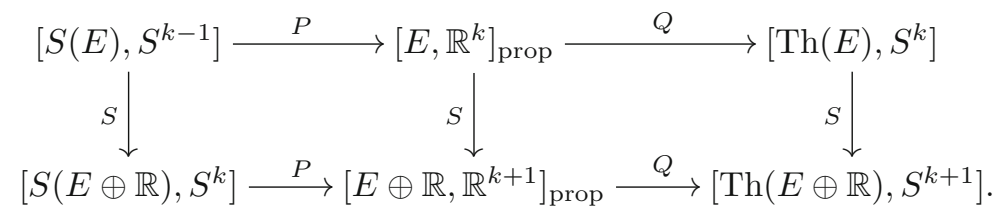

In the proof of Theorem 1.1, we saw that the maps $P$ are bijections. We now investigate when the other maps in the diagram are bijective.

Let us now assume that $k \geq 2$, that $M$ is a finite connected $\mathrm{CW}$-complex of dimension $m$, and that $E$ is a normed real vector bundle of rank $n$. Since $k \geq 2$, based and unbased homotopy classes into $S^{k-1}$ and $S^{k}$ coincide, as well 
as based or unbased proper homotopy classes into $\mathbb{R}^{k}$ and $\mathbb{R}^{k+1}$. We denote by

$$
\pi^{k-1}(S(E)):=\left\langle S(E), S^{k-1}\right\rangle
$$

the $(k-1)$-th cohomotopy set of $S(E)$. We refer to [7, Chapter VII] for information on the cohomotopy sets we use below. The cohomotopy set $\pi^{k-1}(S(E))$ is not always a group, but it is if $m+n \leq 2 k-3$. We investigate the long exact sequence of the pair $(\overline{B(E)}, S(E))$ if $m+n \leq 2 k-3$ :

$$
\pi^{k-1}(\overline{B(E)}) \rightarrow \pi^{k-1}(S(E)) \stackrel{\delta}{\rightarrow} \pi^{k}(\overline{B(E)}, S(E)) \rightarrow \pi^{k}(\overline{B(E)}) .
$$

Since $\overline{B(E)}$ deformation retracts to $M$ and $S^{k-1}$ is $(k-2)$-connected, we see that if $m \leq k-2$, there are isomorphisms

$$
\pi^{k-1}(\overline{B(E)}) \cong \pi^{k-1}(M) \cong 0 \quad \text { and } \quad \pi^{k}(\overline{B(E)}) \cong \pi^{k}(M) \cong 0 .
$$

Thus we conclude that for $2 k \geq m+3+\max (n, m+1)$, there is an isomorphism $\pi^{k-1}(S(E)) \cong \pi^{k}(\overline{B(E)}, S(E))$. The relative cohomotopy set is the cohomotopy set of the quotient for nice spaces, thus $\pi^{k}(\overline{B(E)}, S(E))=$ $\pi^{k}(\overline{B(E)} / S(E))=\pi^{k}(\operatorname{Th}(E))$. The coboundary map is an isomorphism $\pi^{k-1}(S(E)) \cong \pi^{k}(\mathrm{Th}(E))$ in the dimension range. Let us consider the based version of Diagram (1)

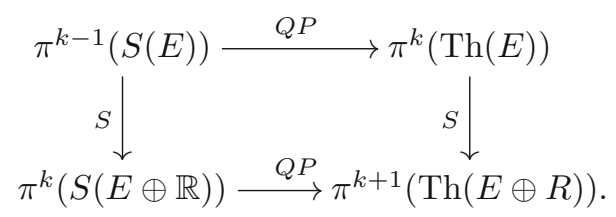

The horizontal maps can be identified with the coboundary map $\delta$ in (2) and therefore the horizontal maps are isomorphisms in the right dimension range. Freudenthal's suspension theorem, cf. [8], states that if $m+n \leq 2 k-2$, the suspension map $\pi^{k}(\operatorname{Th}(E)) \rightarrow \pi^{k+1}(S \operatorname{Th}(E)) \cong \pi^{k+1}(\operatorname{Th}(E \oplus \mathbb{R}))$ is an isomorphism. Combining all this information gives us the following theorem.

Theorem 3.1. Let $M$ be a finite $C W$ complex of dimension $m$ and $E$ a normed real vector bundle over $M$ of rank $n$. Let $k \geq 2$ and suppose that $2 k \geq m+3+$ $\max (n, m+1)$. Then all maps in Diagram (1) are bijections.

This theorem expresses a stability phenomenon: For all $l$ sufficiently large, the map $Q$ induces bijections $\left[E \oplus \mathbb{R}^{l}, \mathbb{R}^{k+l}\right]_{\text {prop }} \rightarrow\left[E \oplus \mathbb{R}^{l+1}, \mathbb{R}^{k+l+1}\right]_{\text {prop }}$. We define the stable homotopy classes of proper maps as

$$
\left[E, \mathbb{R}^{\mathrm{k}}\right]_{\mathrm{prop}}^{S}=\lim _{l \rightarrow \infty}\left[E \oplus \mathbb{R}^{l}, \mathbb{R}^{k+l}\right]_{\text {prop }}
$$

Recall that the stable homotopy and cohomotopy groups of a space $X$ are similarly defined

$$
\pi_{k}^{S}(X)=\lim _{l \rightarrow \infty}\left\langle S^{l} S^{k}, S^{l} X\right\rangle \quad \text { and } \quad \pi_{S}^{k}(X)=\lim _{l \rightarrow \infty}\left\langle S^{l} X, S^{l} S^{k}\right\rangle .
$$

A direct corollary of Theorem 3.1 is then 
Corollary 3.2. Let $M$ be a finite $C W$ complex of dimension $m$ and $E$ a normed real vector bundle of rank $n$. Then $Q$ induces a bijection of $\left[E, \mathbb{R}^{k}\right]_{\text {prop }}^{S}$ with $\pi_{S}^{k}(\operatorname{Th}(E))$.

Stable homotopy and cohomotopy groups are related via Spanier-Whitehead duality, which we recall now. We refer to the original references $[11,12]$ for these statements. Let $i: X \rightarrow S^{N}$ be a sufficiently nice embedding of a sufficiently nice space $X$ into a sphere (e.g. a smooth embedding of a compact manifold, or the inclusion of a CW complex as a subcomplex). Then the space $D_{N} X=S^{N} \backslash i(X)$ is a Spanier-Whitehead dual of $X$. The stable homotopy type of $D_{N} X$ is well defined: It is independent of the dimension $N$ and the choice of embedding. The fundamental result is that $\lim _{l \rightarrow \infty}\left[S^{l} X, S^{l} Y\right]$ is in bijection with $\lim _{l \rightarrow \infty}\left[S^{l} D_{N} Y, S^{l} D_{N} X\right]$. In particular, the stable cohomotopy groups of $X$ are the stable homotopy groups of $D_{N} X$ with a dimension shift. Now let us assume that $M$ is a compact manifold with boundary $\partial M$. There is a unique (up to isotopy) embedding of $M$ into $\mathbb{R}^{m+n}$ for $n$ sufficiently large. Let $E$ be the normal bundle of such an embedding, i.e. let $E$ be the stable normal bundle of $M$. Atiyah [2, Proposition 3.2] showed that $S D_{m+n}(M / \partial M) \simeq \operatorname{Th}(E)$. If the boundary $\partial M$ is empty, we should interpret $M / \partial M$ as $M$ with a disjoint basepoint added. The Spanier-Whitehead dual of a sphere is $D_{n+m} S^{n+m-k}=S^{k-1}$. We have

$$
\begin{aligned}
\pi_{n+m-k}^{S}(M / \partial M) & :=\lim _{l \rightarrow \infty}\left\langle S^{l} S^{n+m-k}, S^{l} M / \partial M\right\rangle \\
& =\lim _{l \rightarrow \infty}\left\langle S^{l} D_{n+m}(M / \partial M), S^{l} D_{n+m} S^{n+m-k}\right\rangle \\
& =\lim _{l \rightarrow \infty}\left\langle S^{l-1} \operatorname{Th}(E), S^{l} S^{k-1}\right\rangle \\
& =\lim _{l \rightarrow \infty}\left\langle S^{l} \operatorname{Th}(E), S^{l} S^{k}\right\rangle \\
& =\pi_{S}^{k}(\operatorname{Th}(E)) .
\end{aligned}
$$

Theorem 3.1 states that $\pi_{S}^{k}(\operatorname{Th}(E))$ is in bijection with $\left[E, \mathbb{R}^{k}\right]_{\text {prop }}^{S}$. We have proven Theorem 1.3.

4. Framed submanifolds and cobordisms. Pontryagin [10] showed that homotopy classes of maps $M \rightarrow S^{k}$, where $M$ is a closed manifold, are in one to one correspondence with framed cobordism classes of $(n-k)$-dimensional manifolds in $M$. Framed cobordism classes are also invariants of homotopy classes of proper maps $E \rightarrow \mathbb{R}^{k}$ but they are not complete, nor is every cobordism classed realized by some proper map. In this section, we discuss this.

4.1. Invariants of proper maps: framed submanifolds and cobordisms. Let $M$ be a smooth $m$-dimensional manifold and $N$ be a connected oriented smooth $k$ dimensional manifold. Every continuous proper map is homotopic as a proper map to a smooth proper map, hence we can consider only proper smooth maps in the proper homotopy classification of proper maps. Suppose that $f: M \rightarrow N$ is a smooth map that is proper. Proper maps between manifolds are closed maps. The set of critical points is closed, hence the set of regular values of a proper map is open. By Sard/Brown's theorem, regular values of 
$f$ are residual, and by Baire's category theorem, it follows that the regular values are dense. Thus the set of regular values of a proper map is open and dense. An example of a smooth function $\mathbb{R}_{>0} \rightarrow \mathbb{R}$ whose set of regular values is not open is $x \mapsto \frac{1}{x(2+\sin (x))}$, but of course this map fails to be proper and closed. The preimage of a regular value $y$ is a closed submanifold $X=f^{-1}(y)$ of dimension $m-k$. Such a manifold can be framed: Let $e_{1}, \ldots, e_{k}$ be a basis of $T_{y} N$ that is compatible with the orientation of $N$. Then for every $x \in X$, the differential of $f$ induces an isomorphism $d f_{x}: N_{x} X \rightarrow T_{y} N$ of the normal space $N_{x} X$ to $X$ at $x$ with $T_{y} N$. Then $\left(\nu_{f}\right)_{x}=\left(\left(d f_{x}\right)^{-1}\left(e_{1}\right), \ldots,\left(d f_{x}\right)^{-1}\left(e_{k}\right)\right)$ is an ordered basis of the normal space $N_{x} X$ at $x$. Letting $x$ vary, this patches together to a map $\nu_{f}$ that trivializes the normal bundle of $X$. The map $\nu_{f}$ is called the framing of $X$. We call $\left(X, \nu_{f}\right)$ a Pontryagin manifold of $f$ and it depends on the choices we made.

Let $F:[0,1] \times M \rightarrow N$ be a homotopy of proper maps between $f_{0}=$ $F(0, \cdot)$ and $f_{1}=F_{1}(1, \cdot)$. By a reparametrization of the homotopy variable, we may assume that $F(t, x)=f_{0}(x)$ and $F(1-t, x)=f_{1}(x)$ for $t$ small. If $y$ is a regular value of the maps $F, f_{0}$, and $f_{1}$ simultaneously, then $(W=$ $\left.F^{-1}(y), \nu_{F}\right)$ is a framed compact submanifold with framed boundary $\left(X_{0}=\right.$ $\left.f_{0}^{-1}(y), \nu_{f_{0}}\right)$ and $\left(X_{1}=f_{0}^{-1}(y), \nu_{f_{1}}\right)$. The framed manifold $\left(W, \nu_{F}\right)$ is a framed cobordism between the framed manifolds $\left(X_{0}, \nu_{f_{0}}\right)$ and $\left(X_{1}, \nu_{f_{1}}\right)$. Being framed cobordant, defines an equivalence relation on the set of framed submanifolds and the framed cobordism class of a Pontryagin manifold of a proper map $f: M \rightarrow N$ does not depend on the choice of the regular value $y$ and the choice of the oriented basis of $T_{y} N$ and is an invariant of the proper homotopy class of $f$. We denote the set of framed $(m-k)$-dimensional closed submanifolds of $M$ up to framed cobordism by $\Omega_{m-k}^{\mathrm{fr}}(M)$.

4.2. The Pontryagin-Thom construction. The framed cobordism class of the preimage of a regular value is in some cases enough to recover the homotopy class of the map: Suppose $M$ is closed and $(X, \nu)$ is a $(m-k)$-dimensional framed submanifold. Out of this data we can construct a (proper) map $f$ : $M \rightarrow S^{k}$, for which $(X, \nu)$ is a Pontryagin manifold: We define $f$ to map $X$ to the northpole $y$ of $S^{k}$ and describe what happens in a tubular neighborhood of $X$. The framing $\nu$ defines, for each point $x \in X$, a diffeomorphism of the normal space around $x$ to a neighborhood of $y$. We use this to extend the map to the tubular neighborhood of $X$ in $M$. One can arrange this in such a way that if one approaches the boundary of the tubular neighborhood, the image under $f$ converges to the south pole. The map $f$ can now be extended to the whole of $M$ by mapping everything outside the tubular neighborhood to the south pole. The northpole is a regular value for $f$ and the Pontryagin manifold at the north pole is exactly the framed manifold $(X, \nu)$. This construction also works for framed cobordisms. This proves the following theorem.

Theorem 4.1. The Pontryagin-Thom construction gives a one to one correspondence between the set $\Omega_{m-k}^{\mathrm{fr}}(M)$ of framed cobordisms in $M$ and the set $\left[M, S^{k}\right]$ of homotopy classes of maps from $M$ to $S^{k}$. 
For more details of the Pontryagin-Thom construction in this classical setting, we refer to Milnor [9] and Pontryagin [10].

\subsection{How good of an invariant is the framed cobordism class of the Pontryagin} manifold of a proper map? A proper map $f: \mathbb{R}^{n} \rightarrow \mathbb{R}^{k}$ is proper homotopic to a map $P g$, where $g: S^{n-1} \rightarrow S^{k-1}$ is a smooth map. The map $P g$ fails to be smooth at the origin, but can be smoothed without affecting the Pontryagin manifolds below. Let $y \in S^{n-1}$ be a regular value of $g$, and $\left(X, \nu_{g}\right)$ be the Pontryagin manifold of $g$ at $y$. The value $y \in S^{k-1} \subseteq \mathbb{R}^{k}$ is also a regular value for the map $P g$. The Pontryagin manifold of $P g$ at $x$ is $\left(X,\left(\nu_{g}, \mu\right)\right)$, where $\mu$ is the last component of the framing which points radially outward from the sphere. So a framed submanifold cannot occur as a Pontryagin manifold if it is not framed cobordant to a framed submanifold that lies on a sphere where the last component of the framing is radially pointing outward.

Let us discuss an explicit example of a framed manifold that does not occur as the Pontryagin manifold of a map. Consider the submanifold $X=\{-1,1\} \subseteq$ $\mathbb{R}$ with framing $\nu_{-1}=\nu_{1}=\frac{\partial}{\partial t}$. Then $(X, \nu)$ cannot occur as the preimage of a regular value of a proper map $f: \mathbb{R} \rightarrow \mathbb{R}$. Suppose on the contrary that such a map exists with $f(-1)=f(1)$. From the framing and the definition of the derivative, we see that there exists an $\epsilon>0$ such that $f(-1+\epsilon)>f(-1)$ and $f(1-\epsilon)<f(1)$. The intermediate value theorem then gives the existence of another point $-1+\epsilon<p<1-\epsilon$ such that $f(p)=f(-1)=f(1)$. Hence $f^{-1}(f(1)) \neq X$ and we conclude that there does not exist a proper $f: \mathbb{R} \rightarrow \mathbb{R}$ with $(X, \nu)$ as a Pontryagin manifold.

But there are also framed submanifolds which are framed cobordant to a framed submanifold which is contained in the unit sphere and has a framing with last component pointing radially outward which do not arise from proper maps. To see this, consider the manifold $X=\{-2,-1,1,2\}$ with framing $\nu(-2)=\nu(2)=\frac{\partial}{\partial t}$ and $\nu(-1)=\nu(1)=-\frac{\partial}{\partial t}$. Then $(X, \nu)$ is framed cobordant to the empty set, however it cannot occur as the Pontryagin manifold of a proper map: If $y$ is the regular value for which $(X, \nu)$ is hypothetically the Pontryagin manifold at $y$, there must be a point $x \in(-1,1)$ such that $f(x)=y$ by the same reasoning as above. The framed submanifold $(X, \nu)$ does not occur as the Pontryagin manifold of a proper map, but the empty set, which is framed cobordant to it, does.

Finally we discuss the fact that the invariant is not complete. The maps $f, g: \mathbb{R} \rightarrow \mathbb{R}$ given by $f(x)=x^{2}$ and $g(x)=-x^{2}$ are not proper homotopic. However as the maps are not surjective the framed cobordism class of both maps is the empty manifold. Hence the framed cobordism class of a regular value cannot distinguish these maps. Here is a more complicated example: Let $f, g: S^{3} \rightarrow S^{2}$ be the Hopf map and the Hopf map precomposed with a degree -1 map of $S^{3}$ respectively. These maps represent +1 and -1 in $\pi_{3}\left(S^{2}\right) \cong$ $\mathbb{Z}$ and are not homotopic. By Theorem 1.1, $P f$ and $P g$ are therefore not proper homotopic, however their Pontryagin manifolds are framed cobordant. To see this, note that $Q P f$ and $Q P g$ are the suspensions of $f$ and $g$ and the Pontryagin manifolds of $Q P f$ and $Q P g$ can be identified with those of 
$P f$ and $P g$. The suspension map $S: \pi_{3}\left(S^{2}\right) \cong \mathbb{Z} \rightarrow \pi_{4}\left(S^{3}\right) \cong \mathbb{Z} / 2 \mathbb{Z}$ is the reduction modulo 2. Therefore the maps $Q P f$ and $Q P g$ are homotopic, so their Pontryagin manifolds must be framed cobordant. But this implies that the Pontryagin manifolds of $P F$ and $P G$ are framed cobordant by general position.

4.4. Open manifolds and the Pontryagin-Thom construction. In Corollary 3.2, we have seen that the homotopy classes of proper maps out of vector bundles stabilize. We expect that if $M$ is an arbitrary open manifold, the homotopy classes of proper maps $\left[M \times \mathbb{R}^{l}, \mathbb{R}^{k+l}\right]_{\text {prop }}$ stabilize when $l \rightarrow \infty$. This suggests that there is a stable Pontryagin-Thom construction for proper maps. A framed submanifold $(X, \nu) \in \Omega_{m-k}^{\mathrm{fr}}(M)$ gives rise to a framed submanifold $((X, 0), \nu \oplus \mu) \in \Omega_{m-k}^{\mathrm{fr}}\left(M \times \mathbb{R}^{l}\right)$ via stabilization. The framing $\nu \oplus \mu$ extends the framing $\nu$ with a fixed basis $\mu$ of $\mathbb{R}^{l}$. Since we expect that the homotopy classes of proper maps stabilize, we also expect that there is a well defined stable bijective Pontryagin-Thom construction $\Omega_{m-k}^{\mathrm{fr}}\left(M \times \mathbb{R}^{l}\right) \rightarrow\left[M \times \mathbb{R}^{l}, \mathbb{R}^{l+k}\right]_{\text {prop }}$ for $l$ sufficiently large.

Acknowledgements. I would like to thank Alberto Abbondandolo, Hansjörg Geiges, Gijs Heuts, and Federica Pasquotto for discussions on the content of this paper. This research was supported by NWA startimpuls - 400.17.608.

Open Access. This article is distributed under the terms of the Creative Commons Attribution 4.0 International License (http://creativecommons.org/licenses/ by $/ 4.0 /$ ), which permits unrestricted use, distribution, and reproduction in any medium, provided you give appropriate credit to the original author(s) and the source, provide a link to the Creative Commons license, and indicate if changes were made.

Publisher's Note Springer Nature remains neutral with regard to jurisdictional claims in published maps and institutional affiliations.

\section{References}

[1] Abbondandolo, A., Rot, T.O.: On the homotopy classification of proper Fredholm maps into a Hilbert space. J. Reine Angew. Math. (2018) (to appear)

[2] Atiyah, M.F.: Thom complexes. In: Proceedings of the London Mathematical Society. Third Series, vol. 11(1), pp 291-310 (1961)

[3] Elworthy, K.D., Tromba, A.J.: Differential structures and Fredholm maps on Banach manifolds. In: Global Analysis (Proceedings of Symposia in Pure Mathematics, vol. XV, Berkeley, CA, 1968), pp. 45-94. American Mathematical Society, Providence, RI (1970)

[4] Gęsba, K.: Fredholm $\sigma$-proper maps of Banach spaces. Fund. Math. 64(3), 341373 (1969)

[5] Geiges, H.: Isotopies vis-à-vis level-preserving embeddings. Arch. Math. 110(2), 197-200 (2018)

[6] Hatcher, A.: Algebraic Topology. Cambridge University Press, Cambridge (2002) 
[7] Hu, S.T.: Homotopy theory. In: Pure and Applied Mathematics, vol. VIII. Academic Press, New York (1959)

[8] Kochman, S.O.: Bordism, stable homotopy and Adams spectral sequences. In: Fields Institute Monographs, vol. 7. American Mathematical Society, Providence, RI (1996)

[9] Milnor, J.W.: Topology From the Differentiable Viewpoint, p. ix +65. Princeton University Press, Princeton (1965)

[10] Pontryagin, L.S.: Smooth manifolds and their applications in homotopy theory. In: American Mathematical Society Translations, Ser. 2, vol. 11, pp. 1-114. American Mathematical Society, Providence, RI (1959)

[11] Spanier, E.H.: Duality and S-theory. Bull. Am. Math. Soc. 62(3), 194-203 (1956)

[12] Spanier, E.H., J.H.C., Whitehead: Duality in homotopy theory. Mathematika. J. Pure Appl. Math. 2, 56-80 (1955)

Thomas O. Rot

Vrije Universiteit Amsterdam

Amsterdam

The Netherlands

e-mail: t.o.rot@vu.nl

Received: 8 March 2019 